\title{
Ambivalences du risque
}

\section{David Le Breton*}

\section{Résumé}

L'objectif de cet article est de rappeler l'ambivalence et la complexité de la notion de risque. L'existence individuelle oscille entre vulnérabilité et sécurité, risque et prudence. Parce que l'existence n'est jamais donnée par avance dans son déroulement le goût de vivre l'accompagne et rappelle la saveur de toute chose. Ne pas prendre de risque n'en est pas moins un risque, celui de la sclérose, de l'engluement dans les routines. C'est se condamner à ne jamais transformer les choses même si elles ne sont pas les meilleures, par exemple se maintenir dans un état de sujétion ou de mal être, être impuissant à s'inventer.

Mots-clés : risque, aventure, sécurité, prudence, ambivalence, identité.

\section{Ambivalences of the risk}

\section{Abstract}

The purpose of this article is to recall the ambivalence and complexity of the notion of risk. Individual existence oscillates between vulnerability and security, risk and prudence. Because existence is never given in advance, it is accompanied by the taste of life that recalls the flavour of everything. Do not take a risk is no less a risk, that of being stiffened, swallowed by routine. It is to doom oneself to never changing things even if they are not the best ones - for example to stay in a state of subjection or misery, to be powerless to invent oneself.

Keywords: risk, adventure, security, caution, ambivalence, identity.

\footnotetext{
*Université de Strasbourg, Strasbourg, France.
} 
«Chaque fois qu'il va trop loin, c'est qu'il n'est pas allé assez loin »

Elias Canetti (Le territoire de l'homme, 1978).

\section{Le risque inhérent à la condition humaine}

u regard des générations antérieures nous vivons dans des conditions
sécurisées à de multiples égards, à travers un lien social moins marqué
de violence, même si les incivilités sont souvent pointées dans certains lieux. Les avancées de l'hygiène et de la médecine, I'instauration de la sécurité sociale, la protection du travail ou les législations innombrables sur les technologies collectives, les règlementations entourant toutes les pratiques sociales, l'élévation du niveau de vie, l'augmentation considérable de la longévité ces dernières décennies l'attestent. Mais ce qui reste de danger et de violence est devenu intolérable. Les sociétés les plus « sécurisées », celles qui vivent dans le plus grand confort, sont les plus inquiètes. Plus s'accroissent les protections et plus la sécurité est au cœur des projets politiques, et plus les individus craignent pour leur vie (Castel, 2003, p. 6). Les chiffres portant sur les indicateurs de santé ou de sécurité ne rassurent pas sur le sentiment personnel de fragilité ni sur l'état de la planète. Au regard de la vie quotidienne nous ne vivons pas dans un monde plus dangereux qu'autrefois, même si les menaces majeures sont devenues colossales, pourtant, au-delà des statistiques rassurantes, apparaissent des menaces d'une nature radicalement différentes que celles affrontées par les générations antérieures (risque nucléaire) ou, à une autre échelle, le risque terroriste dans un contexte de mondialisation. D'autres sources d'inquiétude s'accroissent du fait de la pollution grandissante de la planète dont les conséquences vont du réchauffement du climat à une altération infinitésimale sans doute mais nocive avec le temps de l'alimentation. Les Inuit du Groenland et de Sibérie, pourtant éloignés des lieux de production ou d'usage des produits chimiques, possèdent un taux de mercure élevé 
dans le sang, et le lait des mères Inuits est saturé de toxiques : « La raison en est que l'alimentation inuit de base se compose de baleines, de phoques et d'oiseaux de mer qui mangent du poisson, des mollusques et des crevettes. Or les produits chimiques se concentrent plus à chaque étape de la chaine alimentaire» (Diamond, 2006, p. 782). «Personne ne peut plus s'échapper nulle part », écrit Kundera (1986, p. 22). La sécurité est impensable en un seul lieu, en une seule époque, le monde est tout entier relié pour le meilleur ou pour le pire, et toute personne engluée à son tour dans cet enchevêtrement sur lequel elle n'a qu'une prise limitée.

La question du risque est désormais au cœur de nos sociétés et de I'existence individuelle. Les individus vivent dans un univers de sens et non dans un monde régi par les statistiques. L'individualisation de nos sociétés détache des anciennes solidarités, induit un sentiment d'isolement, marqué par un sentiment accru de fragilité. La personne prend une importance croissante, elle fait presque un monde à elle seule et craint toujours d'être le point d'imputation du désastre. Tout inattendu prend dès lors la forme d'un désaveu cruel dont il faut trouver les responsables. Mais I'obsession d'anticiper et de parer toute déconvenue se heurte à l'opacité du monde et à l'imprévisibilité d'évènements enchevêtrés dans une multitude de causalités réciproques. La puissance d'agir et de comprendre de l'individu est infiniment limitée pour nombre de faits de la vie courante, et elle entretient la peur. Plus on cherche à se protéger plus on se rend sensible à ce qui échappe à toute prise, et plus le sentiment de vulnérabilité s'accroit. Le risque est désormais synonyme de menace, on oublie qu'il est parfois la source même de l'exaltation de soi, de l'intensité d'exister. A l'aune de la vie courante, le risque est souvent vécu comme une donnée négative venant menacer un équilibre antérieur. S'il n'est pas la conséquence d'un choix, il est plutôt une mauvaise surprise, une menace rôdant autour de la sphère privée, une altérité échappant à tout contrôle.

L'existence est à la fois assurée et fragile, toujours quelque peu sur le fil du rasoir, l'individu est voué à une part d'incertitude. Chaque jour dévoile son lot inégal d'événements attendus et de surprises. Le chemin parait de prime abord tout tracé, mais l'inattendu l'emporte parfois. Le matin ignore ce que réserve le soir. La condition affective et sociale n'est 
jamais donnée une fois pour toutes, elle impose un débat permanent avec les autres, avec les choses, au risque d'en être meurtri. L'existence n'est pas ciselée dans la calme évidence de son aboutissement comme un fil tendu au cordeau enjambant les difficultés du terrain. Elle est plutôt sinuosités du chemin, ambivalences. Elle est propre à engager sur des voies que rien ne laissait présager. L'individualisation du lien social, la personnalisation des significations et des valeurs induit l'éloignement des autres, avec les protections qu'ils étaient susceptibles d'offrir. Vivre côte-à-côte, voire même dans la concurrence ou l'indifférence, dans l'inquiétude des différences, n'est pas vivre ensemble.

Sans l'exercice d'une certaine prudence la vie serait impossible ou brève. Une connaissance intuitive des risques de l'environnement, une vigilance diluée dans les faits et gestes du quotidien permettent de se prémunir. Mais ce tissu de précautions intégrées dans les habitudes de vie s'adosse en permanence à des menaces potentielles. La vie la plus tranquille n'est jamais immunisée contre l'inattendu qui prend la forme pour le meilleur ou pour le pire, de la maladie, de l'accident ou de l'ennui, de soucis professionnels, du licenciement, de l'échec d'une entreprise, de séparations affectives, de deuils, ou de rencontres, de moments d'émerveillements, de découvertes, de création, etc. Elle n'est pas à l'abri du désir irrépressible qui naît d'une image saisie au fil d'un récit ou d'une discussion, ou de l'exquise tyrannie de l'occasion qui arrache à soi-même sans laisser à la réflexion le temps de revenir sur sa décision. La confiance élémentaire envers les autres ou le monde est parfois rompue de manière inouïe. Les nuages toxiques ou les eaux contaminées rejetées dans la mer portent leur menace de mort sur des milliers de kilomètres, et sans que son irruption soit repérable car elle est invisible, inodore, intangible, sauf à travers des technologies fines. Le danger n'est plus enfermé dans les frontières d'un Etat, il est désormais de partout et de toujours, il déborde toute limite nationale, il touche des zones considérables s'agissant par exemple des technologies contemporaines comme le nucléaire, ou encore de pandémies... Mais il prend aussi la figure d'individus nomades qui subvertissent la confiance nécessaire au lien social pour introduire l'épouvante au cœur des lieux les plus paisibles à travers des entreprises terroristes par exemple. Et dès lors prendre un café avec 
des amis à la terrasse d'un café ou regardez un feu d'artifice sur le front de mer d'une grande ville peut s'avérer une décision tragique quand les tueurs font irruption. L'affaire de la vache folle met dans les assiettes les plus ordinaires des germes de la maladie. Dans les années quatre-vingt, le sida inscrit le danger au cœur des relations sociales les plus heureuses et les moins suspectes. L'affaire du sang contaminé transforme certains médecins et administrateurs en pourvoyeurs de maladie et de mort. Des médicaments largement diffusés sur le marché se révèlent porteurs de dangers pour les patients (médiator par exemple). Le péril vient parfois de l'environnement et s'impose au corps défendant de l'individu emporté dans un univers qui se défait : tremblement de terre, glissement de terrain, inondation, tsunami, tempête, effondrement, incendie, accident d'une centrale nucléaire... Un moment de distraction en traversant la rue, l'infarctus ou l'agression, l'épidémie, plongent dans une fatalité malaisée à prévoir ou à retourner en sa faveur. L'heureuse rencontre amoureuse d'une nuit peut se transformer quelques mois plus tard en séropositivité ou en séparation tragique. Nul ne chemine au sein d'un bunker, protégé contre le risque même de vivre.

\section{Ambivalence du risque et de la sécurité}

Les instruments du confort se retournent parfois en sources de danger et sèment la mort ou les blessures (électrocution, explosion, asphyxie, etc.). Pour les terroristes les moyens de transport ordinaires comme des avions de ligne ou des trains, des métros, se transforment en engins de destruction massive. Leur force est de miser sur l'impensable en brisant la confiance qui régit nos existences. Les lieux de convivialité, d'échanges, ceux où toute vigilance est relâchée, sont les cibles privilégiées de leurs actions, les marchés, les restaurants, les cafés, les lieux de culte, etc. Personne ne porte sur son visage un projet de meurtre ou d'attentat, ni même dans son histoire si l'on en juge par nombre de biographies de jeunes djihadistes convertis ces dernières années (Jeffrey et al., 2016 ; Le Breton, 2018). Le terroriste ou le tueur est invisible, mêlé à la foule. Il brouille la frontière entre champs de bataille et populations civiles et répand l'épouvante pour atteindre chacun au cœur de son sentiment de confiance ontologique envers le monde. En visant des hommes et des femmes ordinaires, au cœur 
d'espaces de civilités courants, il dit que nul ne sera épargné, que n'importe qui peut périr, qu'aucun abri n'existe plus même en restant cloitré chez soi. Il est inattendu, imprévisible et d'une extrême cruauté. La circonspection est neutralisée. La suspicion frappe toute situation insolite, toute personne qui échappe à l'ordinaire, et même les personnes les plus ordinaires en viennent à être suspectées. Parfois les instruments de prévention sont à la source même de l'épouvante, en témoigne le crash de l'avion allemand de la Germanwings puisque le co-pilote s'était enfermé dans le cockpit dans l'impossibilité pour le commandant de bord et son équipage de I'ouvrir. Après les attentats du 11 septembre 2001, une telle mesure visait en effet à empêcher le détournement d'avion par des pirates de l'air ou des terroristes. La porte du cockpit est verrouillée afin que nul ne puisse l'ouvrir de l'extérieur, même sous la contrainte d'une arme. Un tel dispositif a procuré au co-pilote les conditions idéales pour s'emparer de l'appareil en l'absence du commandant de bord. Les exigences même de sécurité ont provoqué la tragédie. La malveillance est désormais une source vive de la peur. Sans confiance le monde devient imprévisible et menaçant, c'est ce mur invisible qui procurait autrefois une sécurité intérieure que brisent les actions terroristes.

Le risque prolifère pour soi à cause des autres, mais il est aussi présent pour les autres à cause de soi, chacun est pour d'autres un danger potentiel. Ne serait que dans le regard des autres, l'incertitude loge dans le moindre de nos désirs, elle hante toute décision, tout choix. Elle est au cœur de la condition humaine, elle est la rançon du fait que chaque individu crée à chaque instant sa liberté avec une lucidité inégale mais parfois aussi avec une adversité inattendue impossible à prendre en compte avant qu'elle ne survienne. Autrui est toujours une limite, pour le meilleur ou pour le pire. Au-delà de la lucidité du moment et de la volonté individuelle, les circonstances modifient en cours de route l'issue d'une action. Invisible sur le moment la menace est inscrite dans la durée et elle peut surgir un jour ou l'autre. Au sein de l'enchevêtrement des données qui participent du processus social, culturel, relationnel ou personnel, l'individu identifie celles qu'il reconnait afin d'étayer ses décisions. Il escompte ne pas se tromper dans son appréciation des événements mais le monde n'est pas 
I'extension complaisante de ses pensées, et le met parfois radicalement en question. Toute décision parie sur l'avenir. Face à elle s'étend l'immensité du temps qui vient avec une multitude de données en interaction, toujours en mouvement qui se propagent et entrent en collision, ou se confirment, les répercussions en sont seulement imaginées sur le moment, mais dès lors qu'elle prend effet elle entre dans l'immaitrisable et l'aléatoire. Le monde est toujours fait de plus d'imprévisible que de probable. Une fois la décision prise les autres avenirs possibles s'effacent ou deviennent plus difficiles d'accès en cas de repentir. Les conséquences d'un acte n'ont pas toujours la transparence de la décision qui l'a enclenché. Nul ne sait au départ si une décision est bonne, ni même si l'échec ou l'accident ne recèlent pas une chance inespérée, ou à l'inverse, si le succès rencontré provisoirement n'est pas la promesse du pire. Seul l'avenir connaît la réponse, il n'est pas là au moment de la décision, mais il est conditionné par elle. Tout commencement engage et rend difficile un retour.

\section{Inéluctabilité du risque}

Tout choix est risque. Faire un choix consiste d'abord à éliminer toutes les autres alternatives, occuper une situation revient à renoncer aux autres, dans l'ignorance de ce qui a été perdu ou gagné au moment de la décision. Mais le mouvement de l'existence ne cesse de redéfinir la balance. Nul ne sait de quoi l'avenir sera fait. L'échec douloureux d'un moment alimente peut-être la chance d'un autre inscrit tout d'abord dans le sillage de la déception. Et la chance d'une bonne décision peut être remise en question par une évolution inattendue des conditions initiales. «Finalement, écrit Paul Auster, toute vie n'est rien de plus que la somme de faits aléatoires, une chronique d'intersections dues au hasard, de coups de chance, d'événements fortuits qui ne révèlent que leur propre manque d'intentionnalité » (1988, p. 298). La portée de nos actes nous échappent car ils dessinent une ligne nécessaire dans nos existences mais nous ignorons ce qu'elles auraient été au regard d'autres choix. Certains, faits à la légère, sont lourds de conséquences. Mais d'autres aussi effectués pourtant avec lucidité et un examen attentif des données. Un écart est toujours présent entre l'intention et ses conséquences. On sait combien parfois «l'enfer est 
pavé de bonnes intentions ». La multitude des décisions inhérentes à la trame infinie des interactions autour de soi, même à l'échelle de la vie courante, induit souvent un labyrinthe de causes et d'effets réciproques qui amène nécessairement à une part d'incertitude. Dans Zadig, Voltaire marque bien I'impossibilité de se projeter dans l'avenir. Les actions vertueuses de Zadig se transforment en tragédie. Un sage laisse périr un enfant car il sait que devenu adulte il assassinera ses parents. Son attitude en apparence arbitraire et immorale devient dès lors la seule morale. L'avenir est dangereux pour nombre de projets.

Un apologue chinois évoqué par Jean Grenier (1973) illustre les conséquences imprévisibles en germe dans tout événement et il traduit la naïveté d'un jugement qui se voudrait rigoureux mais reste rivé sur une situation immédiate détachée du temps qui passe. Un vieillard vivant seul avec son fils perd un jour son cheval. Ses voisins viennent le soutenir dans sa peine, mais il les arrête : «Comment savez-vous que c'est un malheur?». En effet, quelques jours plus tard, le cheval revient de lui-même entraînant avec lui plusieurs chevaux sauvages. Ses amis félicitent le vieil homme qui les arrête à nouveau : «Comment savez-vous que c'est une chance? ». En effet, le fils essaie de dresser ces chevaux et se brise bientôt la jambe. A ses voisins éplorés venus le consoler, il répond : «Comment savez-vous que c'est de la malchance ? ». L'année suivante une guerre éclate à laquelle le fils ne peut se rendre étant boiteux (Lie-Tseu) (cf. Le Breton, 2016, p. 4142) L'histoire pourrait se poursuivre à l'infini. La décision la plus risquée au départ est parfois la plus judicieuse, soit par une évaluation fine de la situation, soit par un changement inattendu des circonstances qui rend propice une décision au départ préjudiciable. Et celle qui paraissait la plus tranquille peut se révéler une chausse-trappe redoutable.

Dans les Cahiers de Malte Laurids Brigge, Rilke raconte l'émergence de ce qu'il nomme le Terrible, cette irruption qui engloutie toute évidence, foudroyant une sécurité désormais confrontée à l'érosion des frontières de l'individu et du monde : " Le moindre mouvement, et déjà le regard plonge au-delà des choses connues et amies, et le contour, tout à l'heure consolateur, se précise comme un rebord de terreur » (1995, p. 70). Malte chemine dans les rues de Paris et il entre dans un café. A la place qu'il occupe 
d'habitude un homme est assis. Il passe son chemin pour s'asseoir ailleurs, mais il découvre soudain que cet homme est saisi d'effroi. La peur l'envahit à son tour, et il s'enfuit du café. Plus tard, à l'abri dans sa chambre, il pense à cet inconnu : «Oui, il savait qu'en ce moment il s'éloignait de tout ; pas seulement des hommes. Un instant encore, et tout va perdre son sens, et cette table, et cette tasse, et cette chaise à laquelle il se cramponne, tout le quotidien et le proche va devenir incompréhensible, étranger, et clos. Ainsi, il était là, il attendait que ce fût consommé. Et ne se défendait plus » (1995, p. 51). La vie quotidienne multiplie les occasions de péril par choix, distraction, oubli, négligence, méconnaissance du milieu, ou maladresse des autres. À tout moment il importe de s'éloigner ou de se méfier de sources potentielles de dangers physiques. Au fil de l'existence, selon la spécificité des situations, le temps qui passe, les peurs se modifient, et le paysage des risques change. Les peurs d'un adolescent ne sont pas celles du vieil homme, celles d'une femme ne sont pas celles d'un homme, celles du chômeur ne sont ni celles de l'ouvrier ni celles de l'entrepreneur ...

L'existence humaine compose avec les sinuosités du chemin, l'incertitude du comportement des autres, les bonnes ou les mauvaises rencontres, etc. L'ambivalence amène parfois à des comportements défavorables ou à des décisions malheureuses. Les circonstances, les choix opérés, le contexte général expose l'individu à des périls qu'il n'a pas toujours pressenti ou qu'il se croyait à tort ou à raison en mesure d'affronter. Une part d'imprévisible trame en permanence toute existence. Lors des premières années de la vie, l'enfant assimile les précautions élémentaires pour ne pas être affecté par les dangers de son environnement familier. Par l'éducation reçue, les conseils prodigués, et les petits incidents auxquels il est confronté, il apprend à son corps défendant à maintenir une distance propice et une attention moins distraite envers les aspérités inhérentes à sa condition. II apprend à marcher, à nager, à faire du vélo, du patin à roulettes, à se méfier de la circulation routière dans son quartier, etc. II intègre des compétences physiques et réflexives l'amenant au fil du temps à mieux identifier ses ressources, même si le risque de les surévaluer demeure toujours. II apprend à dominer ses peurs et à contrôler les dangers, à assumer les risques symboliques qui touchent à son identité, et notamment à son identité de genre. La vie en 
société exige pour chacun d'être rompu aux précautions pour ne pas être atteint par les événements extérieurs. Elle implique une dialectique entre prudence et risque, entre sécurité et insécurité, une pesée des conséquences des décisions ou des actions.

\section{Jubilation du risque}

Si le risque est imposé ou subi il participe plutôt de la violence, voire même du traumatisme. Dans la vie quotidienne, et au-delà dans les activités technologiques ou scientifiques, économiques ou politiques, le risque est associé plutôt au danger, mais s'il est librement choisi, alors, à l'inverse, il est perçu comme motif d'épanouissement, occasion de se mesurer à une situation inédite, ressource pour redéfinir son existence, éprouver ses capacités personnelles, rehausser l'estime de soi, ou obtenir la reconnaissance des autres. Le risque délibérément pris est une école de caractère. En lui se loge une mobilisation des ressources intimes, une intensité d'existence, une attente passionnée. II est un outil propice pour bouleverser la fixité des choses, les positions établies, ouvrir de nouvelles pistes. « Nous misons tout sur la chance hasardeuse, sur le destin et sur l'à peu près, mais nous détruisons les ponts derrière nous, nous entrons dans le brouillard comme si le chemin devait nous soutenir en toutes circonstances » (Simmel, 1989, p. 314).

Cette donnée anthropologique ne cesse de s'étendre dans une société où I'individu est de plus renvoyé à lui-même comme foyer de sens et de valeur de son existence. La multiplication des activités physiques et sportives à risque va de pair avec une société où, pour un nombre grandissant de nos contemporains vivre ne suffit plus. Il faut éprouver le fait de son existence, expérimenter les sensations de soi, et faire ses preuves pour décider de la valeur de son existence. Ces activités, apparues dans les années quatre-vingt, connaissent un vif succès en termes de participation : sport-aventure, raids, courses d'orientation, marathons, courses ultra, parcours de natation en longue distance, triathlons, tri-triathlons, chute libre, rafting, canyoning, trekking, alpinisme, escalade, parapente, courses d'endurance, défis de toutes sortes ... Plus nos sociétés se protègent par des procédures sécuritaires et diabolisent le risque, et plus grandit la tentation de le solliciter. Régulièrement 
les medias se font les échos complaisants de ce qui devient depuis une vingtaine d'années la routine d'exploits ou d'aventures paradoxales ( premières » spectaculaires, souvent dérisoires par ailleurs, en montagne sur les mers, dans les déserts, défis improbables dans des environnements dangereux, tout en utilisant toutes les technologies contemporaines et la main d'œuvre locale à bas coût) où le preneur de risque ne cesse de donner de ses nouvelles et de téléphoner à ses proches pour les rassurer. Le programme des conférences à leur retour est déjà établi. Ils sont bardés de dispositifs technologiques et leur action financée par des sponsors. Mais le jeu avec le risque est présent, il transforme ces « expéditions » en nouveau spectacle de l'aventure et en mise en valeur narcissique de soi (Le Breton, 2017). Par procuration, il donne le frisson à ceux qui les suivent à distance par écrans interposés et s'identifient à eux.

Mais, souvent de manière plus modeste, sans aller chercher les caméras, en se mettant à l'épreuve l'individu se donne des repères pour produire son identité. Dans l'affrontement physique au monde, il cherche ses marques, s'efforce de tenir entre les mains une existence qui lui échappe. Les limites de fait prennent alors la place des limites de sens qui ne parviennent plus à s'instaurer. En s'affrontant au pire, il cherche à gagner le meilleur, à convertir sa peur, son épuisement, en détermination du caractère. II vit des moments d'intensité d'être, réenchante son existence par l'emprunt de chemins de traverse où il ne doit sa progression qu'à lui-même. Dans nos sociétés où les repères se fragmentent et se multiplient, le corps est toujours une réserve pour savoir qui l'on est et ce que l'on peut attendre du monde. Dans la douleur, la souffrance, le harassement, l'incertitude du lendemain, I'individu éprouve son existence avec une folle intensité. Le jeu avec le risque donne le sentiment d'échapper à sa condition ancienne, de se remettre pleinement au monde (Le Breton, 2017 ; 2012).

L'ambivalence devant le risque est également présente ici. La volonté de sécuriser certains lieux ou certaines pratiques se heurte de plus en plus, d'une part, à l'indifférence de certains usagers, revendiquant une liberté radicale d'agir selon leur volonté et de satisfaire leurs désirs, et, de l'autre, à la méconnaissance des lois ou des conseils, la négligence des panneaux affichant les dangers. Les menaces sont perçues comme telles pour les autres 
mais pas pour soi. Le goût de la transgression ou l'ignorance des consignes de sécurité se conjuguent aujourd'hui pour que des pratiques dangereuses pour soi ou pour les autres se déroulent couramment en mer ou en haute montagne. L'individu se considère souverain quant à son existence et à la mise en œuvre de sa volonté, parfois il en arrive à penser que l'Etat ne doit pas intervenir en la matière.

Dans La loterie à Babylone (1951), Borges observe que les loteries sans probabilité de danger ne rencontraient pas de succès. Elles s'adressaient seulement à l'espoir, leurs concepteurs eurent l'idée d'introduire des éléments défavorables en les mêlant aux récompenses. Chaque billet de cette loterie gratuite, obligatoire et secrète double l'existence, voire la régit toute entière, apportant son lot d'avantages ou de pénalités. Un monde sans risque serait un monde sans aléa, sans aspérités, et livré à l'ennui. Hypothèse cependant impensable car dès lors qu'un vivant existe, il est projeté dans les incertitudes de son milieu, et plus encore I'humain à qui les circonstances imposent des choix innombrables dont les conséquences restent toujours à venir. Si les autres ne sont pas nécessairement l'enfer pensé par Sartre, ils introduisent inéluctablement de l'imprévu. La projection tranquille dans la longue durée, avec l'assurance que rien jamais ne changera, que toute surprise est exclue, suscite l'indifférence, à défaut d'obstacles donnant à l'individu l'occasion de se mesurer à son existence. Se sentir vivant implique d'éprouver parfois le frisson du réel. La rançon possible de la sécurité est la fadeur. À l'inverse, l'établissement dans le danger, s'il s'impose à son corps défendant à l'individu, est rarement une condition heureuse, investie avec passion, il engendre la peur, l'anxiété devant l'irruption probable du pire.

La riposte à la précarité relative de la vie consiste justement dans cet attachement à un monde dont la jouissance est mesurée. Seul a de prix ce qui peut être perdu et la vie n'est jamais acquise une fois pour toutes comme une totalité close et assurée d'elle-même. De surcroit la sécurité étouffe la découverte d'une existence toujours en partie dérobée et qui ne prend conscience de soi que dans l'échange parfois inattendu avec le monde. Le danger inhérent à la vie consiste sans doute à ne jamais se mettre en jeu, à se fondre dans une routine sans aspérité, sans chercher à inventer ni dans son rapport au monde, ni dans sa relation aux autres. Ainsi, ni la sécurité 
ni le risque ne sont des modes d'épanouissement et de création de soi. Le goût de vivre engage une dialectique entre risque et sécurité, entre capacité de se mettre en question, de se surprendre, de s'inventer, et celle de rester fidèle à l'essentiel de ses valeurs ou de ses structures d'identité. Parce que nous avons la possibilité de la perdre, l'existence est digne de valeur (Le Breton, 2017 ; 2016). «Quand j'évoque certains moments périlleux de mon existence, je me les rappelle avec une sorte de joie ; non qu'ils aient comporté une joyeuse surexcitation, mais parce qu'ils m'ont procuré une sensation inédite -une nature nouvelle, pour ainsi dire- et m'ont soulevé momentanément au-dessus de moi-même », écrit par exemple l'écrivain chilien W. Hudson (2002, p. 209).

L'existence individuelle oscille entre vulnérabilité et sécurité, risque et prudence. Parce que l'existence n'est jamais donnée par avance dans son déroulement le goût de vivre l'accompagne et rappelle la saveur de toute chose. Ne pas prendre de risque n'en est pas moins un risque, celui de la sclérose, de l'engluement dans les routines. C'est se condamner à ne jamais transformer les choses même si elles ne sont pas les meilleures, par exemple se maintenir dans un état de sujétion ou de mal être, être impuissant à s'inventer. La «morne incuriosité » dont parle Baudelaire protège sans doute mais elle amène à mourir d'ennui. La prise de risque dans la vie courante est une tentative de redéfinition de l'existence. Certes, la possibilité de perdre demeure, mais elle est peu de chose au regard de la satisfaction d'avoir osé et de ce qu'il est loisible de gagner, ne serait-ce qu'en terme d'estime de soi. En outre, le moment du risque, s'il est choisi, est toujours la certitude de connaitre une intensité d'être qui tranche avec l'ordinaire.

L'acquiescement au risque n'exclut d'ailleurs ni le calcul ni la prudence, à l'image de la philosophie d'Aristote (Aubenque, 1963). Le chœur, à la fin de l'Antigone de Sophocle, formule la nécessité du recul avant toute décision : «La prudence est de beaucoup la première condition du bonheur (...) les orgueilleux voient leurs grands mots payés par les grands coups du sort et ce n'est qu'avec les années qu'ils apprennent la prudence ». La distance réflexive avec le monde tient à la conviction qu'une part d'inattendu est toujours prévisible. Celui qui cède à la précipitation témoigne d'une évaluation lacunaire de la situation dont les conséquences ne se font pas 
attendre. La prudence s'impose comme une qualité morale essentielle à I'homme politique ou au citoyen qui assume une responsabilité envers autrui. Elle est la conscience aiguë de ce que toute décision implique des conséquences non souhaitables sur soi et sur les autres.

Nombre de pratiques sociales exposent en permanence au risque de perdre la face et d'être socialement discréditée (Goffman, 1974). Toute recherche scientifique est un pari sur les résultats. Sur le plateau, le comédien court le risque d'oublier une réplique, d'être saisi par le trac ou une inextinguible crise de fou rire au moment le plus dramatique de la pièce, ou simplement de faire une mauvaise prestation. Le présentateur de télévision ou l'homme politique peut commettre un lapsus ou émettre un jugement irréfléchi qui sème le doute sur sa personne. Le livre d'un écrivain est un pari sur la qualité de son œuvre et sur la fidélité de ses lecteurs. Nul n'est à l'abri d'une création mineure ou d'une radicale perte de créativité. Le meilleur artiste est exposé à produire un jour une œuvre susceptible de remettre en cause son statut et sa stature. Tout acte de création soulève le péril non négligeable d'être mal reçu ou d'être simplement de piètre qualité et de provoquer le jugement défavorable, la moquerie ou l'indifférence. II met en danger la réputation personnelle et donc le sentiment d'identité de celui qui s'engage en toute bonne foi dans l'entreprise. Toute prise de risque met à la merci d'une parole, d'un regard, d'un jugement sans complaisance des autres, voire de leur jalousie ou leur rancœur. L'univers relationnel au sein duquel baigne l'individu n'est jamais une donnée acquise, il est toujours sous la menace du malentendu, du conflit, de la déception. Mais dans le même mouvement, cet inachèvement est aussi propice à l'émerveillement de l'instant, à la découverte de soi et des autres.

David Le Breton est Professeur de sociologie à l'université de Strasbourg et membre de I'Institut Universitaire de France.

$\equiv$ david.le.breton@unistra.fr. 


\section{Bibliographie}

1. AUBENQUE, Pierre. La prudence chez Aristote. Paris : PUF, 1963.

2. AUSTER, Paul. La chambre dérobée. Paris : Actes Sud, 1988.

3. BORGES, Jorge L. La loterie de Babylone. In : BORGES, Jorge L. Fictions. Paris : Gallimard, 1951.

4. CANNETTI, Elias. Le territoire de I'homme : Réflexions, 1942-1972. Paris : Albin Michel, 1978.

5. CASTEL, Robert. L'insécurité sociale. Qu'est-ce qu'être protégé ? Paris : Seuil, 2003.

6. DIAMOND, Jared. Effondrement. Paris : Folio, 2006.

7. GOFFMAN, Erving. Les rites d'interaction. Paris : Minuit, 1974.

8. GRENIER, Jean. L'esprit du tao. Paris : Champs-Flammarion, 1973.

9. HUDSON, William H. Un flâneur en Patagonie. Paris : Payot, 2002.

10. JEFFREY, Denis et al. Jeunes et djihadisme. Les conversions interdites. Lyon : Chroniques Sociales, 2016.

11. KUNDERA, Milan. L'art du roman. Paris : Folio/Gallimard, 1986.

12. LE BRETON, David. Passions du risque. Paris : Métailié, 2012.

13. LE BRETON, David. La sociologie du risque. Paris : PUF, 2016.

14. LE BRETON, David. Conduites à risque. Des jeux de mort au jeu de vivre. Paris : PUF, 2017.

15. LE BRETON, David. Jeunes et radicalisation. Bruxelles : Yapaka, 2018.

16. RILKE, Rainer M. Les cahiers de Malte Laurids Brigge. Paris : Seuil, 1995.

17. SIMMEL, Georg. L'aventure. In : SIMMEL, Georg. Philosophie de la modernité. Paris : Payot, 1989. 
